\title{
Synthesis, Properties, and Derivatization of Poly(dihydrogermane): A Gemanium-based Polyethylene Analogue
}

Haoyang Yu, ${ }^{\S}$ Chuyi Ni, ${ }^{\S}$ Alyxandra N. Thiessen, Ziqi Li and Jonathan G. C. Veinot* Department of Chemistry, University of Alberta, Edmonton, Alberta, Canada T6G 2G2

\begin{abstract}
Polygermanes are germanium-based analogues of polyolefins and possess polymer backbones made up catenated Ge atoms. In the present contribution we report the preparation of a stable germanium polyethylene analogue - polydihydrogermane (i.e., $\left.\left(\mathrm{GeH}_{2}\right)_{n}\right)$ - via two straightforward approaches that involve topotactic deintercalation of the CaGe Zintl phase. The resulting $\left(\mathrm{GeH}_{2}\right)_{n}$ possess morphologically dependent chemical and electronic properties and thermally decompose to yield amorphous hydrogenated Ge. We also show that the resulting $\left(\mathrm{GeH}_{2}\right)_{n}$ provide a platform from which functionalized polygermanes can be prepared via thermally-induced hydrogermylation-mediated pendant group substitution. This facile one-step derivatization reaction exploits $\mathrm{Ge}-\mathrm{H}$ reactivity and opens the door to a wide array of tailored functional polygermanes.
\end{abstract}




\section{Introduction.}

Polyethylene $\left(\mathrm{PE} ;\left(\mathrm{CH}_{2}\right)_{n}\right)$ is the prototypical polyolefin and is ubiquitous throughout modern society; its impact can be seen in seemingly unrelated sectors ranging from packaging to construction to communications and more than 21 million metric tonnes were produced in the United States alone in 2018. ${ }^{1}$ The vast utility of PE arises, in part because it is readily prepared in large quantities, exhibits impressive chemical resistance, and it is processable. For silicon- and germanium-based polyolefin analogues the story is very different.

Polymers with backbones made up of catenated silicon (i.e., polysilanes) and germanium (i.e., polygermanes) atoms are far less prevalent. Correspondingly, their uses are not as far reaching; polysilanes are primarily employed as lithography resists ${ }^{2-4}$ and polygermanes largely remaining as research curiosities..$^{5-7}$ The limited scope of impact of these materials arises in part because of complications related to preparation and reactivity. Still, they remain subjects of considerable interest because, unlike PE, their structures afford electrical conductivity that arises from $\sigma$ delocalization of electrons. ${ }^{8-10}$ Furthermore, these electronic properties (in particular band gaps) can be tailored by defining molecule weight, side-group substitution, and/or introducing tensile strain. ${ }^{10-13}$ As such, poly-silanes and germanes exhibit uniquely tunable optical and electrical properties, such as non-linear optical response ${ }^{14}$ and photo-induced electron transfer. ${ }^{15}$ If a Gebased polyethylene analogue (i.e., $\left.\left(\mathrm{GeH}_{2}\right)_{n}\right)$ can be prepared, it is reasonable it will provide additional fundamental insight into polymers made up of catenated non-carbon Group 14 elements 
(i.e., $\mathrm{Si}$ and $\mathrm{Ge}$ ) and their properties, while offering a platform on which a suite of new materials with exquisitely tunable optical and electronic properties can be developed.

Preparing polygermanes can be challenging; short chain oligogermanes have been synthesized from reactions involving Grignard or organolithium reagents with germanium diiodide; ${ }^{10,16,17}$ higher molecular weight linear organopolygermanes are accessible from diorganodichlorogermane precursors via Würtz-type coupling $5,10,14,16$ and electroreductive synthesis. ${ }^{12,18,19}$ Alternative approaches employing $\mathrm{Ru}$ (i.e., $\left(\mathrm{PMe}_{3}\right)_{4} \mathrm{RuMe}_{2}$ or $\left.\mathrm{Ru}\left(\mathrm{PMe}_{3}\right)_{4}\left(\mathrm{GeMe}_{3}\right)_{2}\right)^{17,20}$ catalyzed demethanative coupling of methylgermanes have also been reported and provide inorganic/organic hybrids containing polygermanes or substituted polygermanes in high yields, however it is not unreasonable that catalyst residues would impact (even compromise) material electronic properties. Furthermore, while these reactions offer comparatively high molecular weight polymers, the scope of accessible substituents is limited by the availability of appropriate molecular precursors.

To our knowledge, only two historical reports have appeared that describe preparation of ' $\left(\mathrm{GeH}_{2}\right)_{n}$ '. Reports by Royen et al. ${ }^{21,22}$ suggest ' $\left(\mathrm{GeH}_{2}\right)_{n}$ ' could be topotactically liberated from CaGe. While these early contributions do not provide evidence in the context of modern characterization standards that $\left(\mathrm{GeH}_{2}\right)_{n}$ was realized, they did confirm the presence of Ge-H functionalities that could provide for post-synthesis modification using variations of 
hydrogermylation protocols used to tailor Ge substrates, ${ }^{23}$ nanostructures, ${ }^{24}$ and layered polygermanes. $^{25}$

We have drawn inspiration from these early reports and successfully prepared high quality $\left(\mathrm{GeH}_{2}\right)_{n}$ from $\mathrm{CaGe}$ at a gram-scale. In this contribution we describe two deintercalation approaches that afford $\left(\mathrm{GeH}_{2}\right)_{n}($ Scheme 1) and interrogate the morphology, bonding, structural disorder, optical band gap, as well as thermal stability of the resulting products. In addition, we demonstrate that the $\left(\mathrm{GeH}_{2}\right)_{n}$ products are readily modified via the introduction of pendant alkyl chains by employing thermally-induced hydrogermylation.

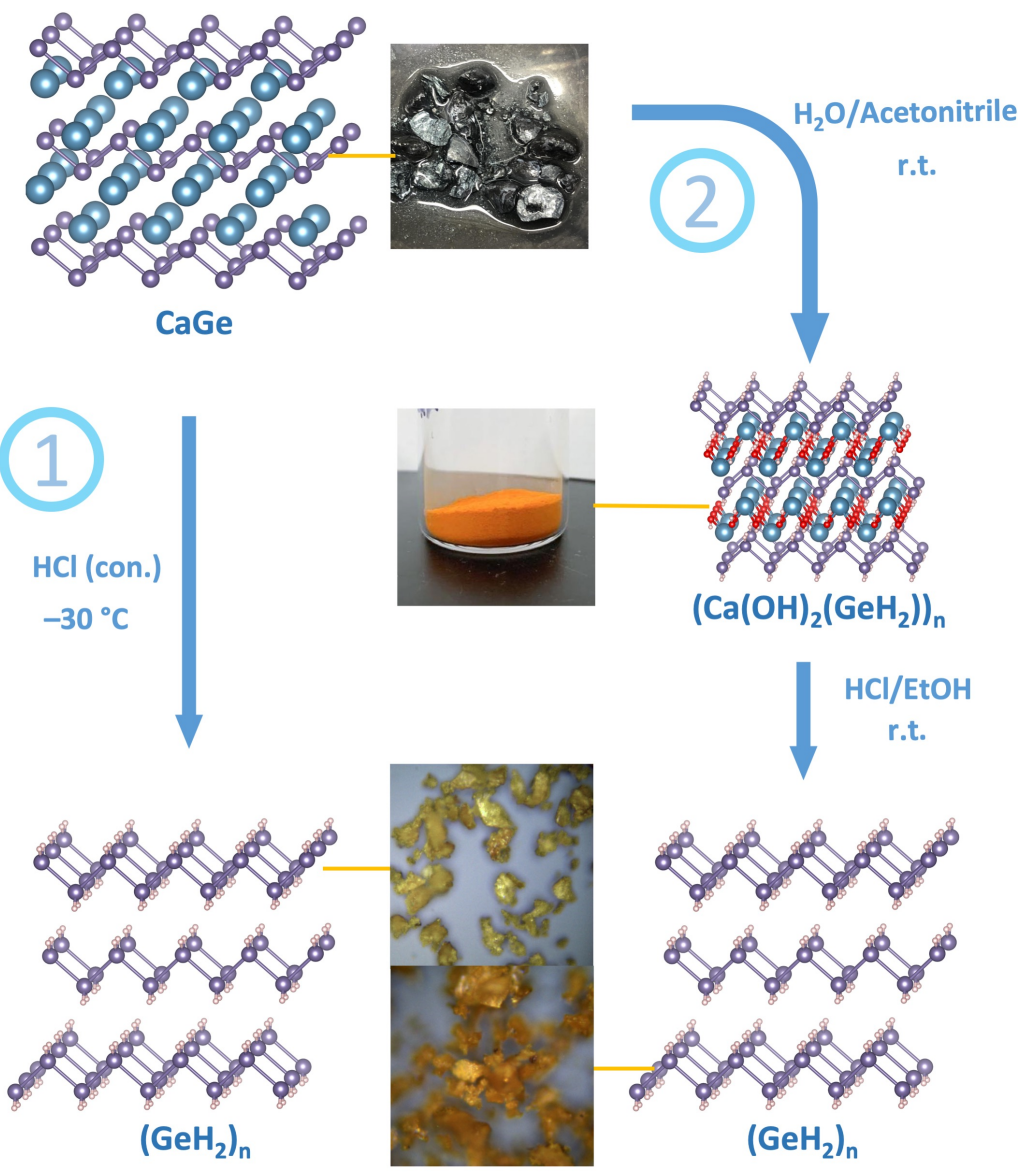

Scheme 1. Two methods for preparing $\left(\mathrm{GeH}_{2}\right)_{n}$ from CaGe. 


\section{Results and Discussion}

Polyhydrogermanes (i.e., $\left.\left(\mathrm{GeH}_{2}\right)_{n}\right)$ investigated in the present study were prepared via two complementary topotactic deintercalation methods from the Zintl phase $\mathrm{CaGe}$ (See: Electronic Supporting Information). CaGe was synthesized by pressing stoichiometric quantities of $\mathrm{Ca}$ and Ge metals into a pellet that was subsequently melted/annealed in an arc-furnace. ${ }^{25,26}$ Powder Xray diffraction (pXRD, Figure 1s) confirmed the as synthesized CaGe is highly crystalline and phase pure. The established crystal structure of $\mathrm{CaGe}$ shows linear chains of bonded germanium atoms in layers that are charge balanced by layers of $\mathrm{Ca}$ ions. ${ }^{27}$ Under ambient conditions $\mathrm{CaGe}$ reacts with moisture (i.e., water) causing it to lose its metallic luster; this process is also accompanied by an obvious color change from grey to light yellow within minutes (Figure S2). The resulting yellow powder takes on a deep orange appearance after several days (Scheme 1). These observations are similar to those noted by Vogg et al. for the reaction of another germaniumbased Zintl phase (i.e., $\left.\mathrm{CaGe}_{2}\right)$ with water that leads to the formation of $\left(\mathrm{Ca}(\mathrm{OH})_{2} \mathrm{GeH}\right)_{n}{ }^{28}$

Our first attempt to prepare $\left(\mathrm{GeH}_{2}\right)_{n}$ drew inspiration from a literature procedure first reported in $1933^{21}$ that is similar to the method employed by us, ${ }^{25}$ and others ${ }^{28-31}$ to exfoliate hydrideterminated germanane from $\mathrm{CaGe}_{2}$ (Method 1, Scheme 1). This process involved direct reaction of $\mathrm{CaGe}$ with cold (i.e., $-30^{\circ} \mathrm{C}$ ) concentrated $\mathrm{HCl} .{ }^{21,27}$ To our surprise, and in stark contrast to the established $\mathrm{CaGe}_{2}$ reaction that is slow and requires days to complete, ${ }^{25,31}$ the reaction with $\mathrm{CaGe}$ is very exothermic and violent causing $\mathrm{CaGe}$ to break down quickly (i.e., within minutes); in fact, 
the entire deintercalation reaction being complete within ca. 30 minutes. Consistent with previous reports, ${ }^{22}$ products of this reaction are not stable when dry and detonate loudly with visible sparks when manipulating $\geq$ ca. $0.25 \mathrm{~g}$ of dry powder under ambient conditions. Similar decomposition was noted for milligram material quantities upon exposure to a standard anti-static gun or 1.99 $\mathrm{mW}$ laser. Clearly, extreme caution must be exercised when performing this reaction and manipulating the corresponding products. Unfortunately, the sensitivity of the cold acid liberated $\left(\mathrm{GeH}_{2}\right)_{n}$ limited comprehensive characterization, however the analyses we could perform provides valuable insight into the material structure and properties.

In light of the violent nature of the direct reaction of $\mathrm{CaGe}$ with cold acid and the sensitivity of the reaction products, we chose to explore alternative methods that could surmount these challenges. Our qualitative observations of trace water (i.e., ambient humidity) induced oxidation of CaGe held the key to developing a new two-step approach for preparing $\left(\mathrm{GeH}_{2}\right)_{n}$ (Method 2, Scheme 1). In this case, CaGe powders were exposed to a solvent mixture of deionized water and acetonitrile to afford an orange solid after approximately $24 \mathrm{~h}$. This intermediate product was isolated by centrifugation and subsequently washed/centrifuged in cold 1:5 v/v HCl/EtOH. The $\left(\mathrm{GeH}_{2}\right)_{n}$ prepared in this way is readily handled as a dry powder under ambient conditions in large (i.e., $\geq 2.0 \mathrm{~g}$ ) quantities, however it does decompose to provide a black solid upon prolonged (i.e., $1 \mathrm{~min}$ ) exposure to a laser (Figure S3)

To investigate the bonding within the products obtained from the two methods described herein we employed Fourier-transform infrared (FTIR), Raman, and X-ray photoelectron 
spectroscopy (XPS) (Figure 1). Consistent with the formation of $\left(\mathrm{GeH}_{2}\right)_{n}$, the FTIR spectrum of the freshly prepared orange product obtained from cold acid deintercalation of CaGe (i.e., Method 1) shows intense $\mathrm{Ge}-\mathrm{H}_{2}$ stretching $\left(2042-2046 \mathrm{~cm}^{-1}\right)$ and bending (ca. 776 and $\left.831 \mathrm{~cm}^{-1}\right)$ modes. ${ }^{30}$ Additional $\mathrm{O}-\mathrm{H}$ stretching and bending features at $\sim 3370$ and $1630 \mathrm{~cm}^{-1}$ are attributed to adsorbed water. ${ }^{25,32}$ For the two-step water oxidation/acid deintercalation process (i.e., Method 2) we first interrogated the orange product obtained from water-induced oxidation of $\mathrm{CaGe}$ and found the FTIR spectrum (Figure S4) showed the abovementioned features associated with $\mathrm{Ge}-\mathrm{H}_{2}$, as well as an additional sharp absorption at $3645 \mathrm{~cm}^{-1}$ that is characteristic $\mathrm{Ca}(\mathrm{OH})_{2}$ stretching - based upon these observations we contend that this material is ' $\left(\mathrm{Ca}(\mathrm{OH})_{2} \mathrm{GeH}_{2}\right)_{n}{ }^{3}{ }^{33}$ Acid treatment of the ' $\left(\mathrm{Ca}(\mathrm{OH})_{2} \mathrm{GeH}_{2}\right)_{n}$ ' provided an orange product that is qualitatively identical (other than stability) to the product recovered from cold acid deintercalation and the FTIR spectrum of this material (Figure 1a) is fully consistent with this proposal. 

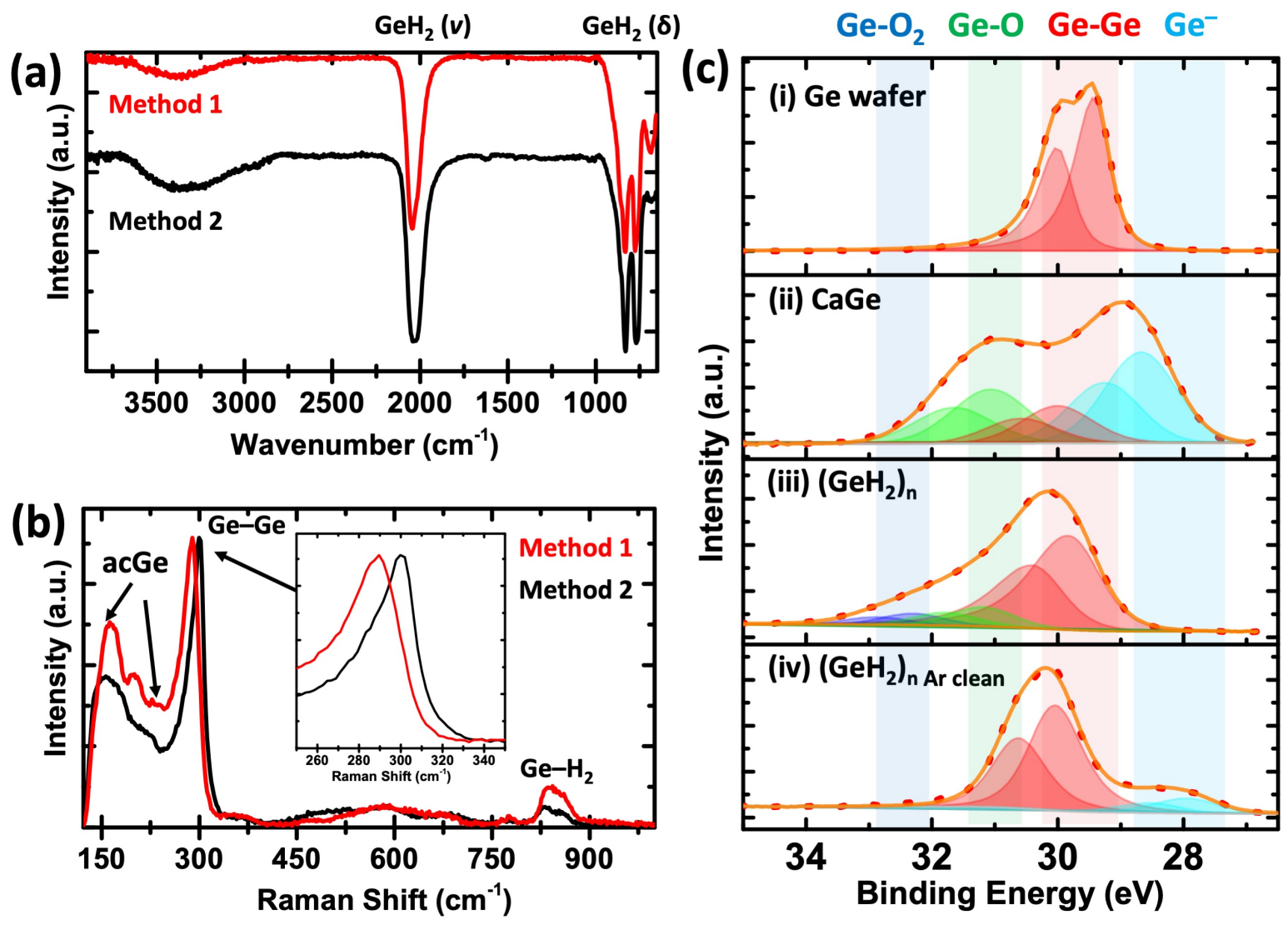

Figure 1. (a) FTIR and (b) Raman spectroscopy of $\left(\mathrm{GeH}_{2}\right)_{n}$ synthesized by Method 1 and 2, inset: highlighting Ge-Ge peak center. (c) High resolution XP spectra of Ge 3d region, (i) Ar plasma cleaned Ge (111) wafer, (b) $\mathrm{CaGe}$ after Ar plasma cleaning, $\left(\mathrm{GeH}_{2}\right)_{n}$ prepared by method 2, (c) before and (d) after Ar plasma cleaning.

Raman spectroscopy provides further support that $\left(\mathrm{GeH}_{2}\right)_{n}$ is being formed with the appearance of an absorption associated with a Ge-Ge optical phonon at 289 and $300 \mathrm{~cm}^{-1}$ for products prepared using Methods 1 and 2, respectively (Figure 1b). Consistent with FTIR analyses, additional $\mathrm{Ge}-\mathrm{H}_{2}$ vibrations are also noted at 180 and $820 \mathrm{~cm}^{-1}$. For $\left(\mathrm{GeH}_{2}\right)_{n}$ prepared using rapid cold acid deintercalation of $\mathrm{CaGe}$, a red-shift in the Ge-Ge optical phonon is observed compared to that of bulk Ge crystal $\left(300 \mathrm{~cm}^{-1}\right) .{ }^{34}$ This observation is reasonably ascribed to a disorderinduced activation of phonon density, as described by Weinstein and Cardona, ${ }^{35}$ and is consistent with our TEM and DRA data (vide infra). In addition to optical phonons, acoustic-like Ge (acGe) 
features at $\sim 160-170 \mathrm{~cm}^{-1}$ and $\sim 225 \mathrm{~cm}^{-1}$ contributed by the vibration of $\left(\mathrm{GeH}_{2}\right)_{n}$ chains were observed,.${ }^{36}$ More intense, blue-shifted acGe peaks were noted in the spectrum of $\left(\mathrm{GeH}_{2}\right)_{n}$ prepared using the two step water oxidation/acid deintercalation approach (Method 2); this difference is reasonably attributed to a closer packing of $\left(\mathrm{GeH}_{2}\right)_{n}$ chains that is also evident in our pXRD analyses (vide infra).

XPS provides insight into the elements present within a given material, as well as their corresponding bonding environments and oxidation states. Unfortunately, this analysis was not possible of the $\left(\mathrm{GeH}_{2}\right)_{n}$ obtained from Method 1 because of its noted sensitivity, however we gained valuable insight into the composition of the product of Method 2. For the present investigation, all spectra were calibrated to adventitious carbon that was set to a binding energy (BE) of $284.8 \mathrm{eV} \cdot{ }^{37,38}$ Survey spectra (Figure S5) show emissions from elements consistent with the expected composition (Note: Evidence of surface oxidation was observed in the spectrum of CaGe (Figure S6)). High-resolution XP spectra of Ge $3 \mathrm{~d}$ region were fit to the $\mathrm{Ge} 3 \mathrm{~d}_{5 / 2}$ and $3 \mathrm{~d}_{3 / 2}$ spin-orbit couple and corresponding couples for different Ge species are presented in Figure 1c as the same color. For CaGe, a low binding energy component centered at $28.62 \mathrm{eV}$ was identified and is a comparable binding energy to $\mathrm{Ge}$ in $\mathrm{CaGe}_{2}$; of important note, this emission appears at a significantly lower binding energy than that of the reference elemental Ge $(29.45 \mathrm{eV}) .{ }^{25} \mathrm{This}$ observation is consistent with the Zintl phase concept that is routinely applied to the understanding of bonding within $\mathrm{CaGe} .{ }^{39,40}$ The XP spectrum of as prepared $\left(\mathrm{GeH}_{2}\right)_{n}$ obtained from Method 2 shows an intense emission at $29.82 \mathrm{eV}$ (Figure 1c(iii)); as expected this is marginally higher than 
the binding energy of $\mathrm{Ge}$ in germanium metal because of the presence of electronegative hydrogen termination. We also observe evidence of trace surface oxidation (i.e., $\mathrm{Ge}-\mathrm{O}(31.1 \mathrm{eV})$ and $\mathrm{Ge}-$ $\left.\mathrm{O}_{2}(32.5 \mathrm{eV})\right)$ that results from sample preparation and manipulation. ${ }^{41}$ All oxygen-based features are removed upon exposure to an Ar plasma and the Ge emission attributed to $\left(\mathrm{GeH}_{2}\right)_{n}$ appears at a $\mathrm{BE}$ of $30.06 \mathrm{eV}$. In addition, while $\mathrm{Ca}$ emissions were not detected in the survey spectrum because they overlap with the Ge LMM signal at ca. $346 \mathrm{eV}$ and Ge $3 \mathrm{~d}$ emission at ca. $30 \mathrm{eV}$, a residual peak centered at $27.90 \mathrm{eV}$ is noted and can only be assigned to $\mathrm{Ge}(-2)$ arising from residual $\mathrm{CaGe}$ that was not deintercalated.

Further insight into the solid-state structure of the present $\left(\mathrm{GeH}_{2}\right)_{n}$ assemblies is obtained from the PXRD patterns of the $\left(\mathrm{GeH}_{2}\right)_{n}$ obtained using synchrotron radiation $(\lambda=0.68745 \AA)$. The patterns from the products of Methods 1 and 2 show broad reflections appearing at ca. 12, 21, 34 and $42^{\circ}$ consistent with short range nanostructured order (Figure 2a). In addition to these reflections, we note low intensity baseline resolved reflections attributable to trace crystalline $\mathrm{CaOCaCl}_{2} \cdot 3 \mathrm{H}_{2} \mathrm{O}$ in the pattern of the $\left(\mathrm{GeH}_{2}\right)_{n}$ obtained from the controlled water oxidation approach (i.e., Method 2). Drawing on past experience with $\mathrm{CaGe}_{2},{ }^{25}$ the observation of broad reflections is consistent with topotactic removal of the calcium ions. In this context, we have indexed the patterns in Figure 2a based upon an orthorhombic unit cell. Doing so reveals that the first two reflections in the pattern correspond to (021) and (200) (Figure S7) where the (200) represents half of the distance between co-planar $\left(\mathrm{GeH}_{2}\right)_{n}$ chains. These dimensions appeared at $3.28 \AA(021)$ and $1.84 \AA$ (200) for the product obtained from cold acid treatment (Method 1) and 
$3.35 \AA(021)$ and $1.87 \AA$ (200) for the product obtained from our two-step water oxidation approach (i.e., Method 2). In both cases, the (021) plane separation approaches that of bulk Ge (111) (3.324 $\AA$ ) while the (200) plane shrinks significantly compare to the parent CaGe $(2.288 \AA) .{ }^{27,42}$ These data suggest the $\mathrm{Ca}$ ions are being removed from $\mathrm{CaGe}$ being replaced by hydrogen atoms; this process results in the $(\mathrm{Ge})_{n}$ chains being ca. $20 \%$ closer to one another (Figure $2 \mathrm{~b}$ ). This structural change disturbs the packing of the $\left(\mathrm{GeH}_{2}\right)_{n}$ chains along the $\mathrm{b}$ axis and no (020) reflection is observed.
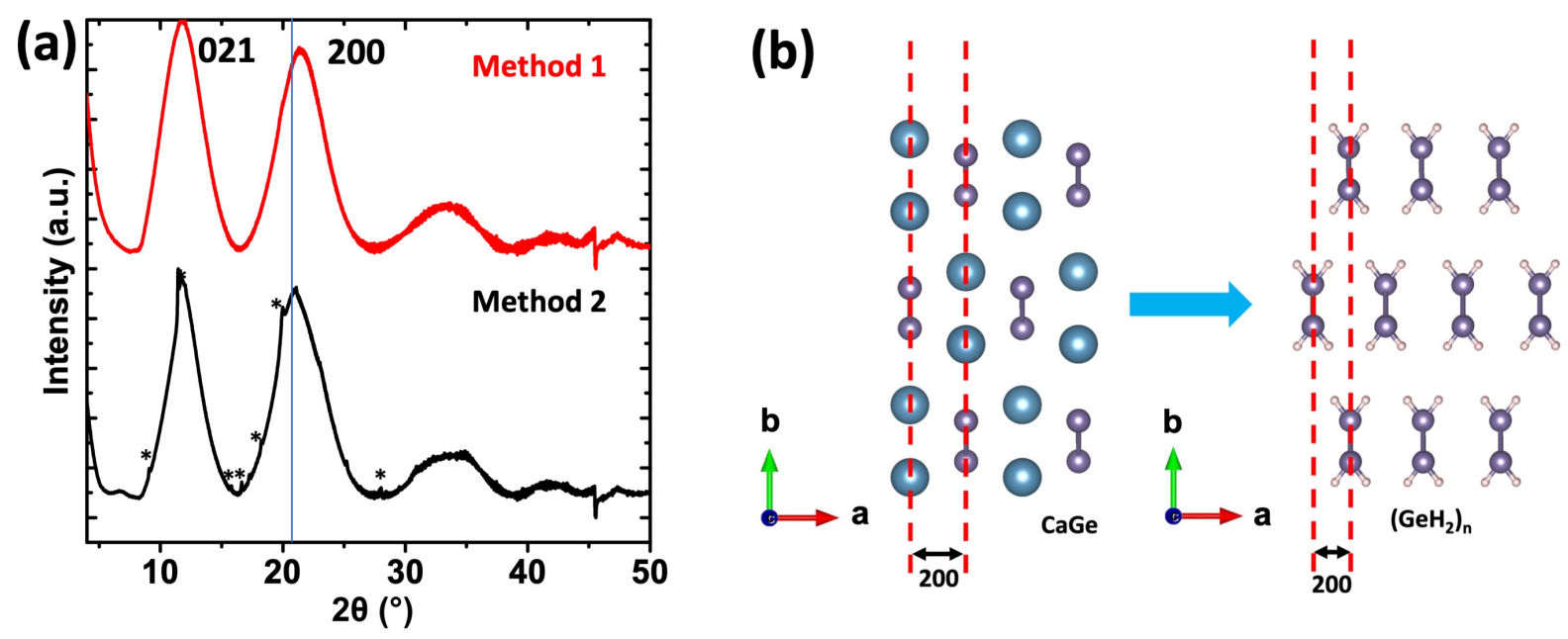

Figure 2 (a) Powder XRD pattern of $\left(\mathrm{GeH}_{2}\right)_{n}$ by two methods, $\lambda=0.68745 \AA$, *trace $\mathrm{CaOCaCl}_{2} \cdot 3 \mathrm{H}_{2} \mathrm{O}$ and (b) Schematic illustration of (200) reflection from (left) $\mathrm{CaGe}$ and (right) $\left(\mathrm{GeH}_{2}\right)_{n}$.

Electron microscopy reveals differences in the morphologies of the two 'types' of $\left(\mathrm{GeH}_{2}\right)_{n}$ that could impact their material properties (Figure 3). Imaging of the $\left(\mathrm{GeH}_{2}\right)_{n}$ provided by Method 1 reveals a random structure while the products of Method 2 shows structural ordering that qualitatively resembles the orientation of ' $\mathrm{Ge}_{n}$ ' chains within the crystalline $\mathrm{CaGe}$ starting material 
and supports the proposal of topotactic extraction of the $\mathrm{Ca}$ ions. It is reasonable this morphological difference reflects the vigorous nature of Method 1 that likely compromises the integrity of the $\left(\mathrm{GeH}_{2}\right)_{n}$ network. The gentler Method 2 preserves the underlying ' $\mathrm{Ge}_{n}$ ' within the parent CaGe.

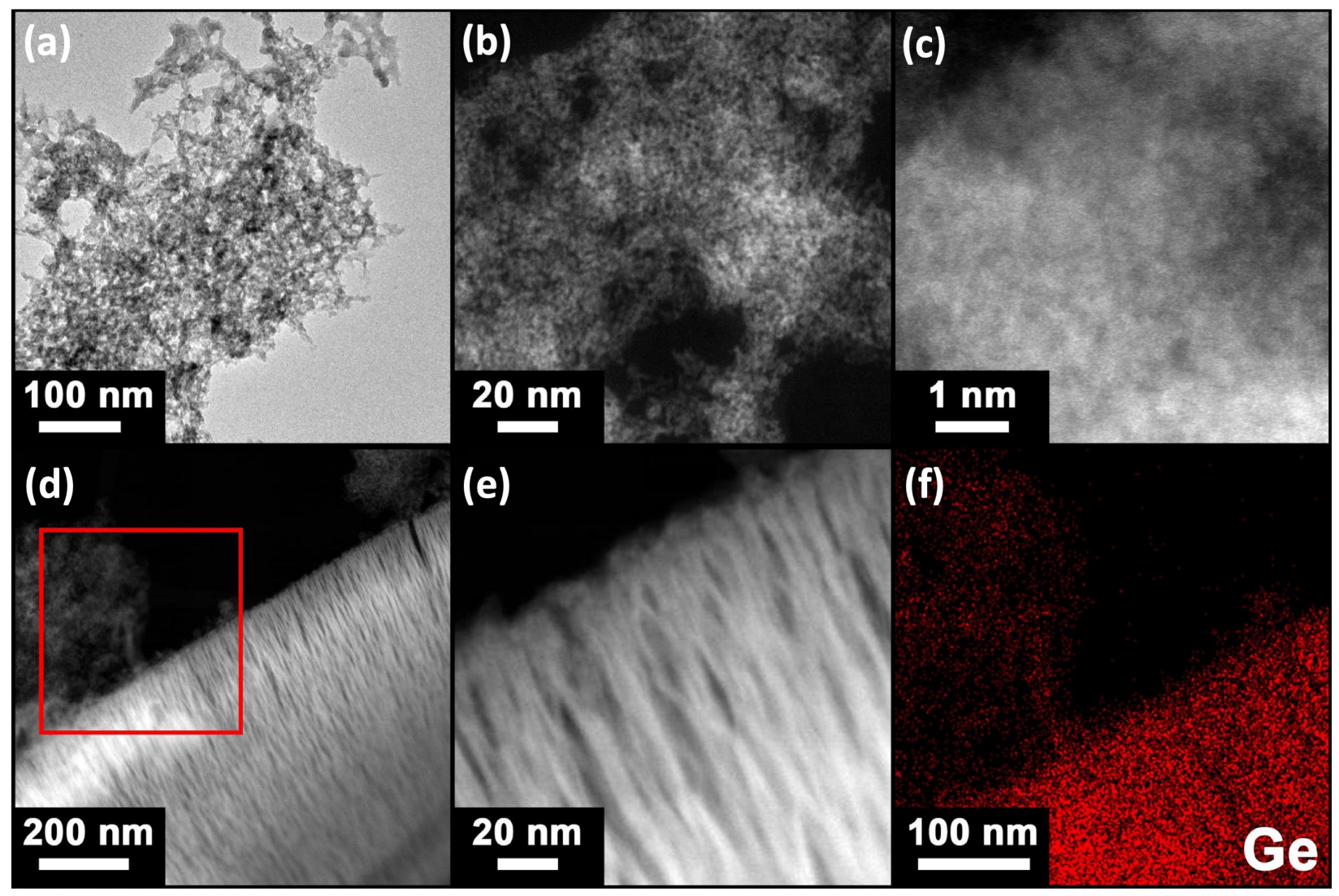

Figure 3. (a-c) TEM analysis of $\left(\mathrm{GeH}_{2}\right)_{n}$ Prepared by Method 1: (d) low magnification TEM image, (e) high magnification and (f) atomic resolution HAADF-STEM images. (a-c) TEM analysis of $\left(\mathrm{GeH}_{2}\right)_{n}$ Prepared by Method 2: (d) low magnification image and (e) high magnification HAADFSTEM images, (f) EDX mapping of Ge $\mathrm{K} \alpha$ at area selected in (d);

Electron microscopy comes with the opportunity to map material composition as a function of morphology using energy dispersive X-ray (EDX) analyses. Consistent with the XPS study presented above, EDX mapping the two $\left(\mathrm{GeH}_{2}\right)_{n}$ morphologies indicates both are comprised primarily of Ge (Figure S8). Furthermore, the EDX spectra are dominated by intense features 
associated with $\mathrm{Ge}$ (i.e., $\mathrm{L} \alpha, \mathrm{L} \beta, \mathrm{K} \alpha, \mathrm{K} \beta$ ) as well as trace $\mathrm{O}$ (ca. $2 \%$ ) and $\mathrm{Cl}$ (ca. $2.5 \%$ ). No Ca is detected at the sensitivity of the EDX method.

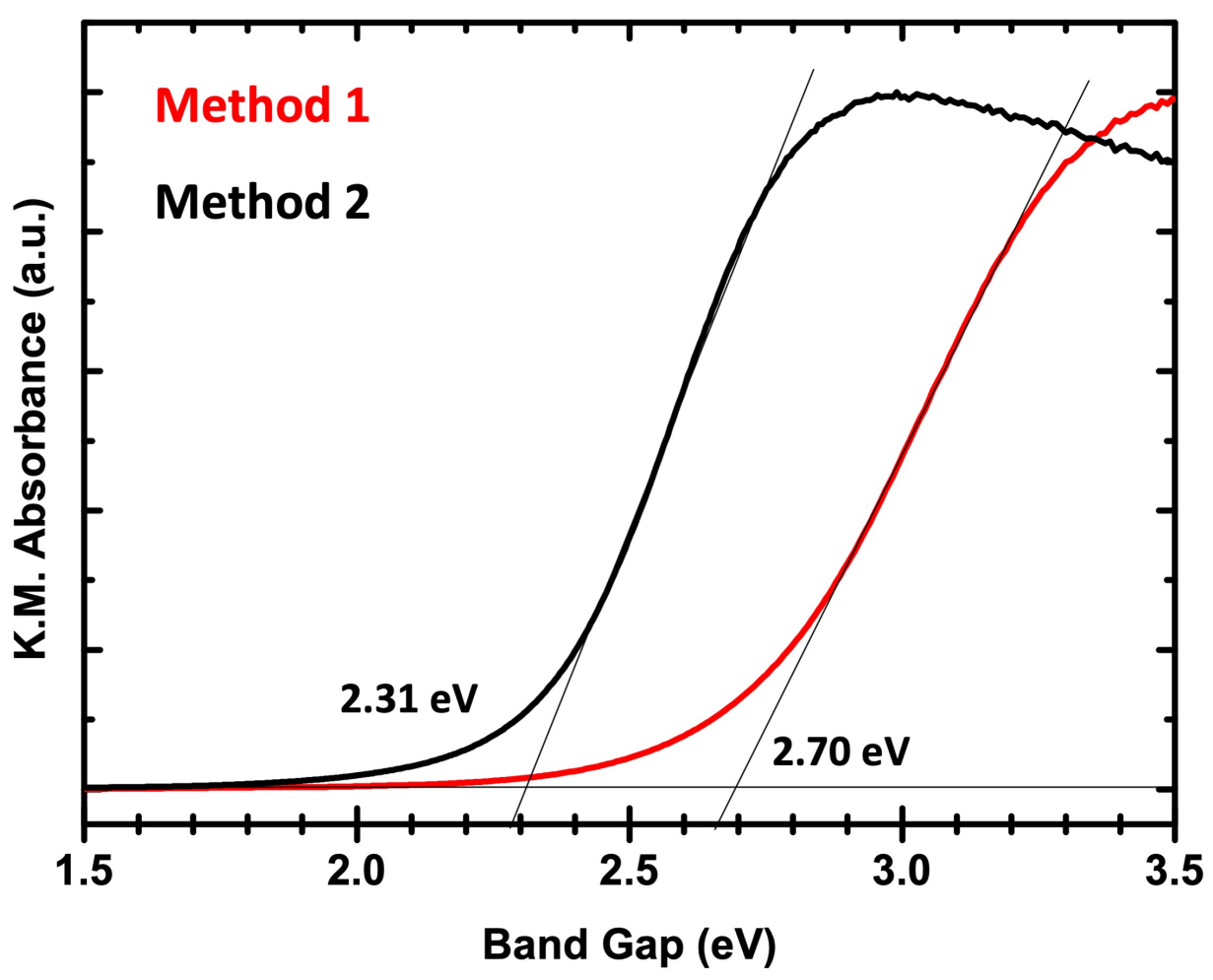

Figure 4. Diffuse reflectance absorption plots for $\left(\mathrm{GeH}_{2}\right)_{n}$ prepared by Method 1 (red) and 2 (black), showing optical band gaps of 2.31 and $2.70 \mathrm{eV}$, respectively.

Having identified the composition and morphology of the $\left(\mathrm{GeH}_{2}\right)_{n}$ obtained from the CaGe deintercalation using Methods 1 and 2, we turned our attention to their respective material properties. Diffuse reflectance absorption (DRA, Figure 4) provides convenient determination of the material optical band gap. For the present $\left(\mathrm{GeH}_{2}\right)_{n}$ samples, optical band gap depends upon mode of preparation and is a reflection of their morphology; optical band gaps of 2.70 and 2.31 eV were obtained for $\left(\mathrm{GeH}_{2}\right)_{n}$ prepared using Methods 1 and 2, respectively. We also note that 
while Urbach tails were observed for both samples, it is more prevalent for $\left(\mathrm{GeH}_{2}\right)_{n}$ prepared using Method 1 and reflects a lack of long range order in the material. ${ }^{30,43}$

The experimental values reported here agree well with first principles calculations reported by Zeng et al. ${ }^{11}$ who predicted a $\left(\mathrm{GeH}_{2}\right)_{n}$ band gap of $3.03 \mathrm{eV}$. These authors indicated that bandedge states in $\left(\mathrm{GeH}_{2}\right)_{n}$ arise primarily from the atomic orbital delocalized over the Ge backbone; in these systems the lowest unoccupied molecular orbital (i.e., LUMO; conduction band edge) arises from Ge $4 \mathrm{~s}$ and $4 \mathrm{p}_{\mathrm{x}}$ orbitals while the highest occupied molecular orbital (i.e., HOMO; valence band edge) is dominated by the Ge $4 \mathrm{p}_{z}$ (along the Ge-Ge chain). Based upon these findings Zeng et al. suggested introducing bulky pendant functionalities (e.g., replacement of Ge-H with Ge-phenyl) would result in negligible impact on the band gap and demonstrated computationally that introducing mechanical (e.g., tensile and compressive) strain provided band gap tuning. ${ }^{11}$ For the present 'real-life' samples, because the $\left(\mathrm{GeH}_{2}\right)_{n}$ are obtained from $\mathrm{CaGe}$ and possess the same pre-existing Ge-Ge skeleton, it is reasonable that the apparent method dependent band gap arises as a consequence differences in the structural strain within the products.

\section{Thermal Stability}

The thermal stability of $\left(\mathrm{GeH}_{2}\right)_{n}$ prepared using Method 2was evaluated using FTIR, Raman spectroscopy and DRA after they had been heated to predefined temperatures in the range of 75 to $200{ }^{\circ} \mathrm{C}$ in an inert environment (i.e., Ar atmosphere and subdued light) for 4 hours (Figures 5 ad). The first, and most immediately striking observation is the noted change in colour. The apparent 
colour of the samples evolved from gold to brown and eventually to black with increased temperature. FTIR and Raman spectra obtained from samples heated at, or below $120{ }^{\circ} \mathrm{C}$ (i.e., magenta traces in Figure 5) show negligible differences from the parent material (i.e., red traces in Figure 5). Increasing the processing temperature to above $120^{\circ} \mathrm{C}$ saw the intensity of $\mathrm{Ge}-\mathrm{H}_{2}$ bending modes in the FTIR spectrum diminish (and eventually disappear) and a broad Ge-O-Ge stretching mode appears that we attribute to material handling. Raman spectra show a similar transition point at ca. $120^{\circ} \mathrm{C}$ at which the Ge-Ge feature red-shifted presumably due to increased structural disorder; this red-shift is accompanied with feature broadened at higher temperatures. The observations made in the FTIR and Raman spectra suggest heating $\left(\mathrm{GeH}_{2}\right)_{n}$ to above $120{ }^{\circ} \mathrm{C}$ induce dehydrogenative reactions that cause the Ge-Ge skeleton to collapse yielding amorphous Ge. Consistent with this proposal we note the optical band gap decreases from $2.31 \mathrm{eV}$ for as prepared samples to $1.50 \mathrm{eV}$ for samples heated at $120{ }^{\circ} \mathrm{C}$. This decrease in band gap is accompanied by an appearance of a more pronounced Urbach tail. Heating at $140{ }^{\circ} \mathrm{C}$ (green line) sees the band gap decrease further to $1.06 \mathrm{eV}$ - a value comparable with amorphous hydrogenated germanium film $(1.1 \mathrm{eV}),{ }^{44}$ and continues to drop to $0.73 \mathrm{eV}$ for sample cured at $200{ }^{\circ} \mathrm{C}$. For samples cured at higher than $140{ }^{\circ} \mathrm{C}$, the Urbach tails become less prevalent suggesting the formation of a more ordered structure.

TGA was performed for $\left(\mathrm{GeH}_{2}\right)_{n}$ prepared by both methods to interrogate their evolution with heating (Figure 5d). A gradual $2 \%$ weight loss was observed for both materials upon heating to $150{ }^{\circ} \mathrm{C}$ that we attribute to the liberation of $\sim 0.75$ eq. of hydrogen. This temperature is lower than 
that observed for layered germanane $\left(200-250{ }^{\circ} \mathrm{C}\right) .{ }^{25,30}$ A second weight loss event (i.e., $16 \%$ and $3 \%$ for $\left(\mathrm{GeH}_{2}\right)_{n}$ from Methods 1 and 2, respectively) was noted between 180 and $220^{\circ} \mathrm{C}$. The 16 $\%$ weight loss noted for Method 1 materials is an order magnitude higher than what would be expected if the entire hydrogen content was liberated from $\left(\mathrm{GeH}_{2}\right)_{n}$. This large weight loss is likely a reflection of the morphological differences between Method 1 and 2 materials noted in our TEM analysis that show Method 1 materials are smaller randomly shaped pieces that would bear more terminal $-\mathrm{GeH}_{3}$ functionalities than the ordered linear structures prepared using Method 2. As such, we attribute this additional weight loss to the liberation of low molecular weight germanes (i.e., $\left.\mathrm{Ge}_{\mathrm{x}} \mathrm{H}_{2 \mathrm{x}+2}\right) .{ }^{45}$ If one reasonably assumes the low molecular weight germanes obtained upon heating Method 1 materials are primarily germane $\left(\mathrm{GeH}_{4}\right)$, the $16 \%$ weight loss corresponds to ca 0.24 eq. of hydrogen. In contrast, for Method 2 materials, which would be expected to contain far fewer terminal groups, the noted $3 \%$ weight loss provides a hydrogen loss of ca. 0.2 eq. and $\mathrm{GeH}_{4}$ loss of ca. 0.02 equivalent. 
(a)
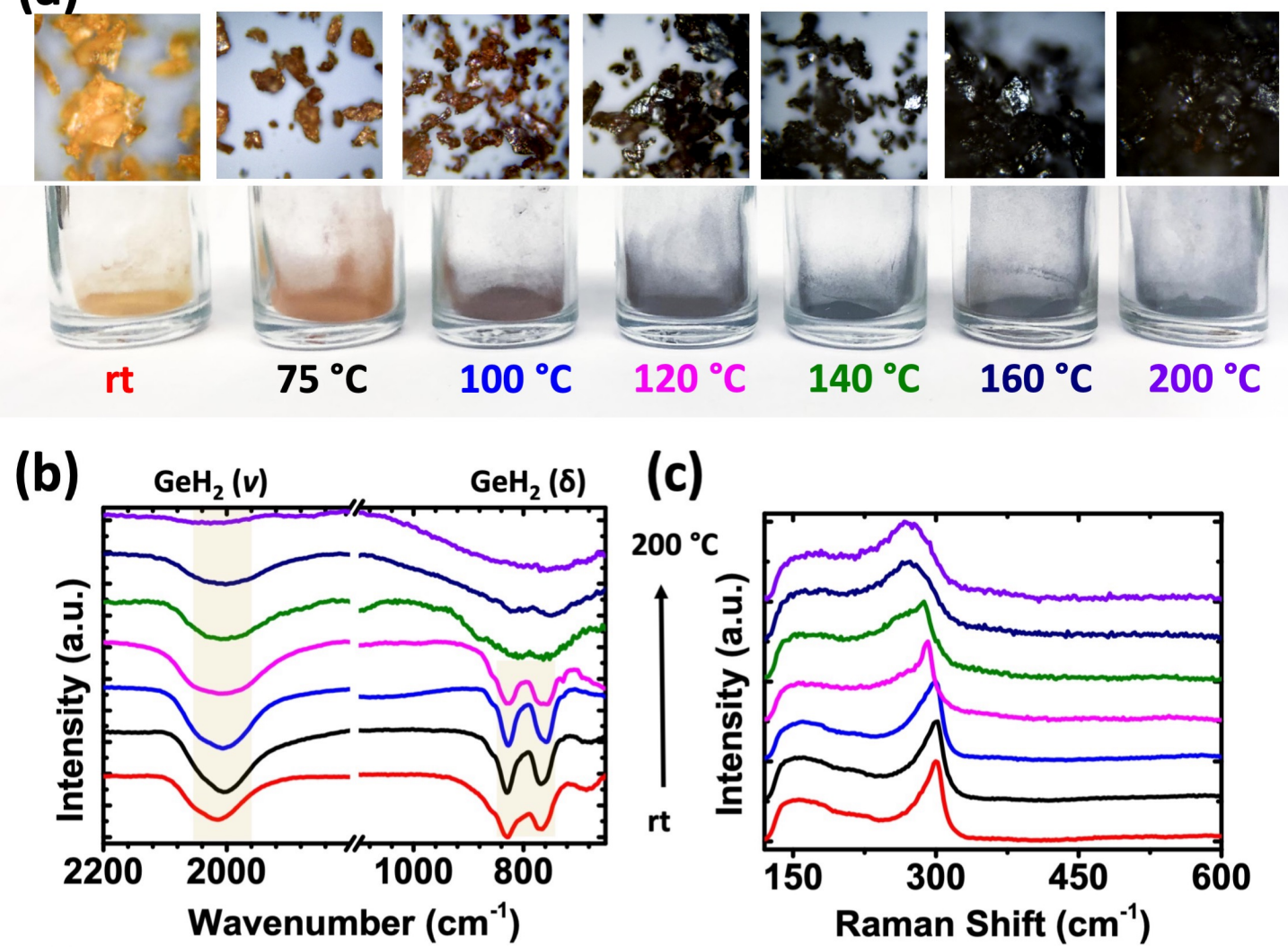

(d)

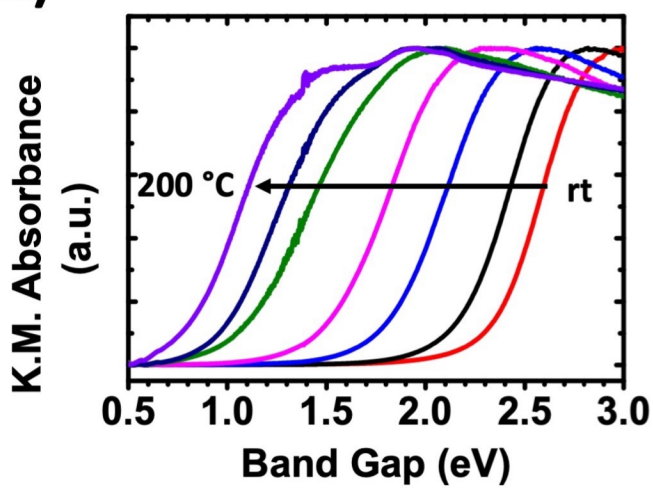

(c)

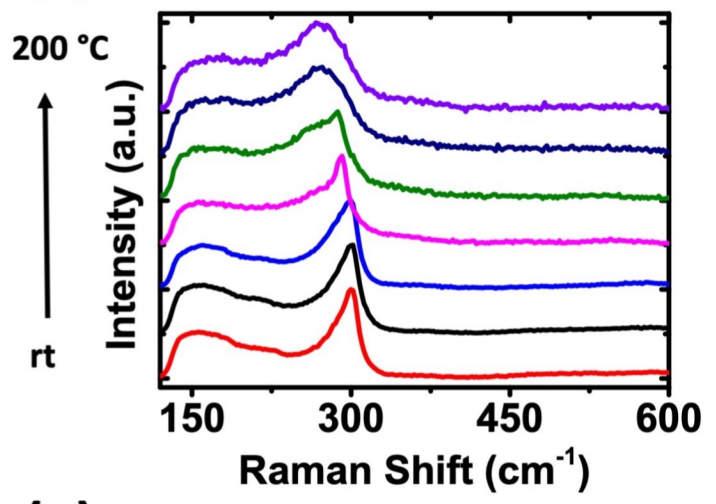

(e)

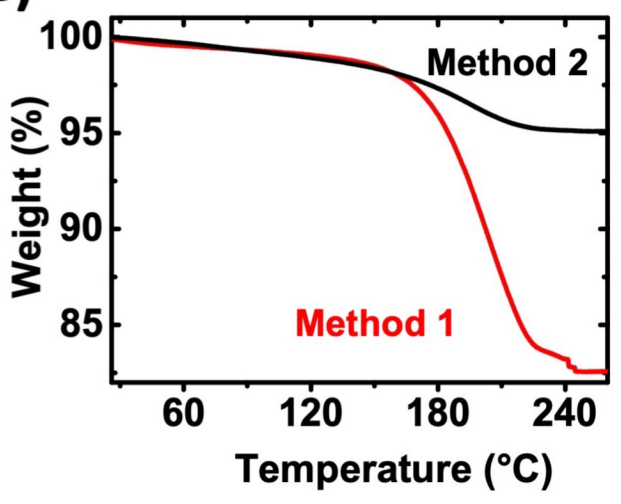

Figure 5. (a-d) $\left(\mathrm{GeH}_{2}\right)_{n}$ annealed at various temperature in Ar environment: (a) optical image, (b) FTIR spectra, (c) Raman spectra and (d) DRA spectra. (e) TGA analysis of $\left(\mathrm{GeH}_{2}\right)_{n}$ prepared by Methods 1 and 2. 


\section{Surface Functionalization}

With $\left(\mathrm{GeH}_{2}\right)_{n}$ in hand, we explored their reactivity and demonstrated side group substitution using thermally-induced hydrogermylation reactions. ${ }^{23,24,46,47}$ Functionalization was achieved by reacting $\left(\mathrm{GeH}_{2}\right)_{n}\left(10 \mathrm{mg}, 0.13 \mathrm{mmol} \mathrm{GeH}_{2}\right)$ and 1-dodecene $(2.0 \mathrm{~mL}, 2.6 \mathrm{mmol})$ in $10 \mathrm{~mL}$ dry toluene. The reaction mixture was subsequently ultrasonicated in a bath sonicator for 3-hours after which it was heated to and maintained at $150{ }^{\circ} \mathrm{C}$ and stirred for 15 hours. Consistent with differing degrees of functionalization, the products obtained from non-sonicated reaction mixtures exhibited limited solvent compatibility while those from sonicated mixtures are compatible with organic solvents and remain in solution for months.

The FTIR spectra (Figure 6) obtained from the reactions involving 1-dodecene with $\left(\mathrm{GeH}_{2}\right)_{n}$ obtained from Methods 1 and 2 were identical and show the expected features of pendant dodecyl groups and no evidence of unsaturated features (i.e., $\mathrm{C}-\mathrm{H} \mathrm{sp}^{2}$ stretching and $\mathrm{C}=\mathrm{C}$ vibrations) of 1dodecene. Consistent with our qualitative observations of solubility, the FTIR spectra products from reaction mixtures that were not sonicated show Ge-H features at ca. 830 and ca. $2010 \mathrm{~cm}^{-1}$ (Figure $6 \mathrm{~b}, \mathrm{c})$. This suggests that in these cases the $\left(\mathrm{GeH}_{2}\right)_{n}$ was incompletely exfoliated and only partially functionalized (i.e., the product corresponds to $\left.\left(\mathrm{GeH}_{\mathrm{x}}\left(\mathrm{C}_{12} \mathrm{H}_{25}\right)_{2-\mathrm{x}}\right)_{n}\right)$. No such $\mathrm{Ge}-\mathrm{H}$ features were evident in the spectra of products from sonicated reaction mixtures. 


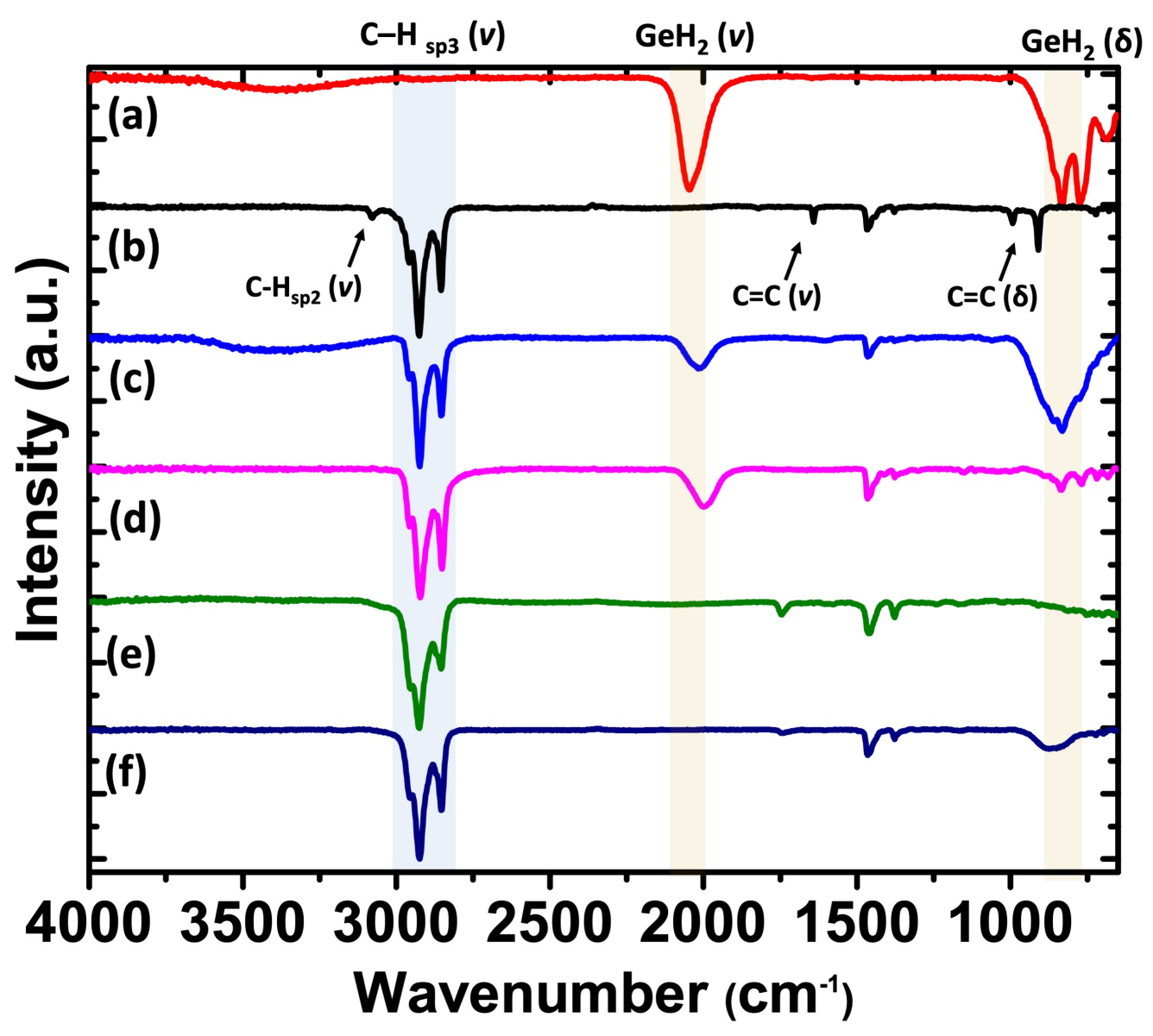

Figure 6. FTIR spectra of (a) $\left(\mathrm{GeH}_{2}\right)_{n}$ prepared by Method 1, (b) neat 1-dodecene, $\left(\mathrm{GeH}_{\mathrm{x}}\left(\mathrm{C}_{12} \mathrm{H}_{25}\right)_{2-}\right.$ $\mathrm{x})_{n}$ prepared without sonication from (c) Method 1 and (d) Method 2, $\left(\mathrm{Ge}\left(\mathrm{C}_{12} \mathrm{H}_{25}\right)_{2}\right)_{n}$ prepared with sonication from (e) Method 1 and (f) Method 2.

EDX mapping of the present $\left.\left(\mathrm{GeH}_{\mathrm{x}}\left(\mathrm{C}_{12} \mathrm{H}_{25}\right)_{2-\mathrm{x}}\right)_{n}\right)$ and $\left(\mathrm{Ge}\left(\mathrm{C}_{12} \mathrm{H}_{25}\right)_{2}\right)_{n}$ confirm $\mathrm{C}$ and $\mathrm{Ge}$ signals are coincident for all samples (Figure $7 \mathrm{c}, \mathrm{d}$ and S9). Consistent with our proposal that sonication is necessary to exfoliate $\left(\mathrm{GeH}_{2}\right)_{n}$ prior to modification via thermally-induced hydrogermylation, HAADF-STEM analyses shows structures of similar morphology to that observed for the parent $\left(\mathrm{GeH}_{2}\right)_{n}$ (Figure 7); taking into consideration our EDX data, this suggests the hydrogermylation reaction may only proceed on the surface of the $\left(\mathrm{GeH}_{2}\right)_{n}$ assemblies. 


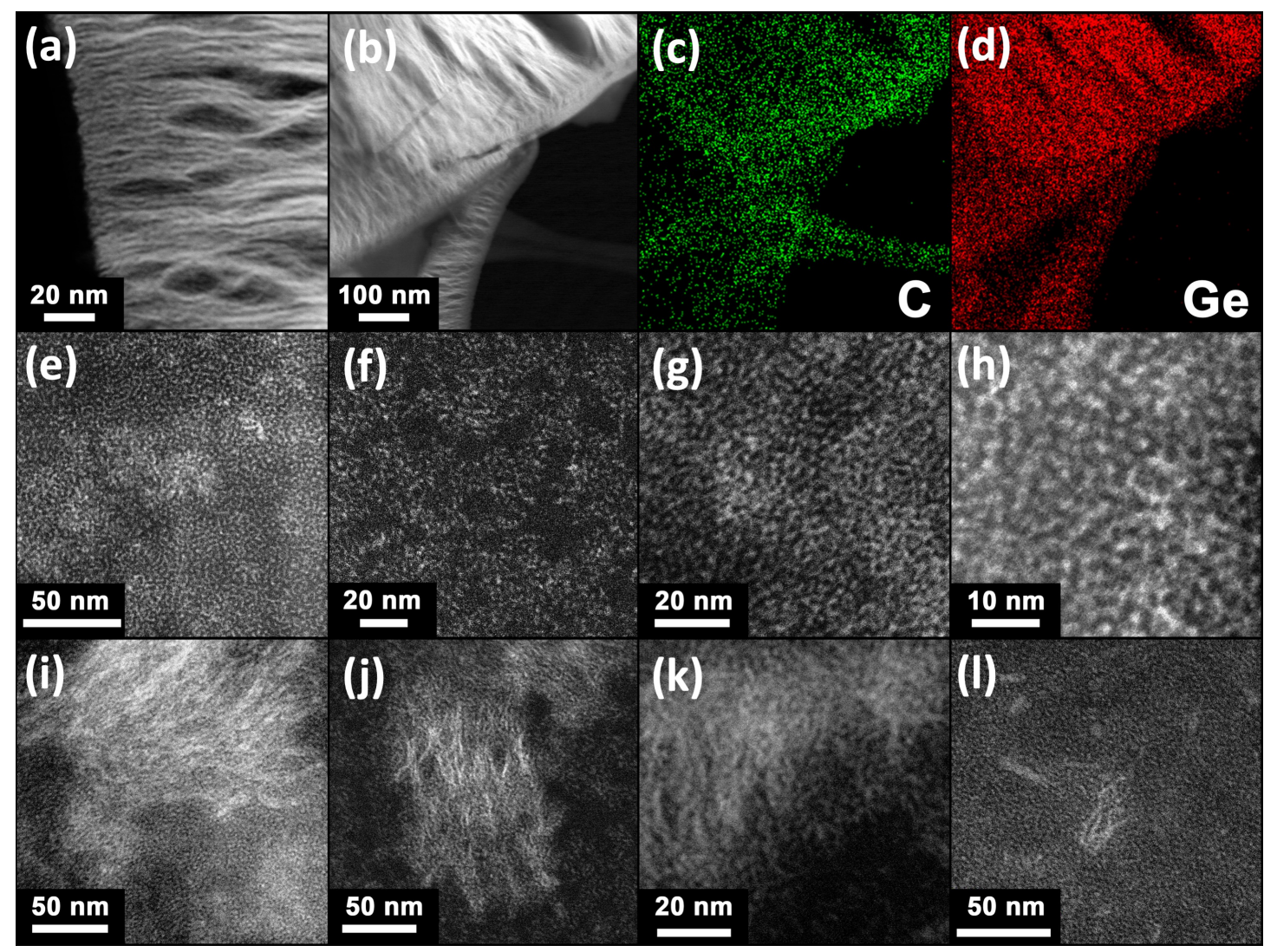

Figure 7. HAADF-STEM images of $\left(\mathrm{Ge}\left(\mathrm{C}_{12} \mathrm{H}_{25}\right)_{2}\right)_{n}$ prepared using Method 2. (a-d) without sonication: (a) high-resolution image, (b) low-resolution image, (c) $\mathrm{C} \mathrm{K \alpha}$ mapping at area (b), and (d) Ge $\mathrm{K} \alpha$ mapping at area (b); (e-h) the assembly of freestanding $\left(\mathrm{Ge}\left(\mathrm{C}_{12} \mathrm{H}_{25}\right)_{2}\right)_{n}$ at different magnification; (i-l) exfoliation process and break down of long Ge-Ge chains.

Evaluation of the products of the sonicated reactions using HAADF-STEM presents a very different picture. Images (Figures 7e-h, S10 c,d) show uniformly distributed patterns on the ultrathin carbon support consistent with the $\left(\mathrm{Ge}\left(\mathrm{C}_{12} \mathrm{H}_{25}\right)_{2}\right)_{n}$ being fully (or near fully exfoliated in solution). We further analyzed the non-fully exfoliated Ge-Ge strands (Figure 7i-1), and noticed the $\left(\mathrm{GeH}_{2}\right)_{\mathrm{n}}$ does not remain a single linear strand. Instead, large fibers will directly decouple into smaller pieces with similar width, explains the narrow distribution for their sizes. Unfortunately, it is challenging to study the fine structure of these materials as images were blurred due to ligand 
coverage, and attempted sample cleaning will alter their morphologies (Figure S11). This will be the basis of future investigations.

Consistent with FTIR and TEM analysis, near identical DRA, Raman, and XP spectra were obtained for products obtained from the functionalization of starting materials obtained from Methods 1 and 2 (Figure S12). This suggests that, despite the parent $\left(\mathrm{GeH}_{2}\right)_{n}$ having qualitatively different structures and stabilities, the $\left(\mathrm{Ge}\left(\mathrm{C}_{12} \mathrm{H}_{25}\right)_{2}\right)_{n}$ the same (at the sensitivity of the methods employed). While straightforward heating of $\left(\mathrm{GeH}_{2}\right)_{\mathrm{n}}$ above $140{ }^{\circ} \mathrm{C}$ provides a material a band gap approaching $1.06 \mathrm{eV},\left(\mathrm{Ge}\left(\mathrm{C}_{12} \mathrm{H}_{25}\right)_{2}\right)_{n}$ heated at the higher temperatures required for hydrogermylation (i.e., $\left.150{ }^{\circ} \mathrm{C}\right)$ and longer times (15 h vs. $\left.4 \mathrm{~h}\right)$ possess a band gap of $2.2 \mathrm{eV}$ (Figure 12a). The is likely due the liquid-solid hydrogermylation reaction between 1-dodecene and $\left(\mathrm{GeH}_{2}\right)_{\mathrm{n}}$ proceeding more rapidly than the solid-solid dehydrogenative reaction of $\left(\mathrm{GeH}_{2}\right)_{n}$ with itself. 


\section{Conclusions}

In summary, we have prepared stable germanium-based polyethylene analog. This polydihydrogermane is comprised of ' $\mathrm{GeH}_{2}$ ' repeat unit and retains a network structure consisting of aligned $\left(\mathrm{GeH}_{2}\right)_{n}$ chains present in the CaGe precursor. XRD shows it has a slightly larger interstrand distance compare with polydihydrogermane prepared in previous reports, which leads to a red-shift in the transverse acoustic vibration in Raman spectroscopy. Thermal stability measurements reveal that this stable form only proceed dehydrogenation reaction slowly with trace $\mathrm{GeH}_{\mathrm{x}}$ decomposition byproducts due to a stronger Ge-Ge bond compared with the traditionally method. Lastly, we show both forms of polydihydrogermane can be modified via hydrogermylation reaction, offering the opportunity for various functional catenated polygermanes.

\section{Associated Content}

Supporting Information.

This material is available free of charge via the internet at http://pubs.acs.org.

PXRD of measured and calculated $\mathrm{CaGe}$, optical photos of $\mathrm{CaGe}$ after exposure in ambient, optical microscopy photo of $\left(\mathrm{GeH}_{2}\right)_{n}$ damaged by laser power, FTIR spectrrum of $\left(\mathrm{Ca}(\mathrm{OH})_{2} \mathrm{GeH}_{2}\right)_{n}$, XPS survey spectra of $\mathrm{Ge}(111), \mathrm{CaGe}$, and $\left(\mathrm{GeH}_{2}\right)_{n}$, high-resolution XP C 1s and Ca 2p spectra of CaGe, structure model of $\left(\mathrm{GeH}_{2}\right)_{n}, \mathrm{EDX}$ spectra of $\left(\mathrm{GeH}_{2}\right)_{n}$ and functionalized polygermane, STEM images of $\left(\mathrm{Ge}\left(\mathrm{C}_{12} \mathrm{H}_{25}\right)_{2}\right)_{n}$, DRA, Raman and XP spectra of functionalized polygermane. 


\section{Author Information}

Corresponding Authors

*Jonathan G. C. Veinot. E-mail: jveinot@ualberta.ca.

Notes

The authors declare no competing financial interest.

\section{ORCID}

Haoyang Yu: 0000-0002-0748-9414

Chuyi Ni: 0000-0002-8032-1685

Alyxandra N. Thiessen: 0000-0001-9978-1422

Ziqi Li: 0000-0002-2296-8114

Jonathan G. C. Veinot: 0000-0001-7511-510X

Author Contributions

$\S \mathrm{H}$. Yu and C. Ni contributed equally to this work.

\section{Acknowledgment}

The authors recognize continued generous funding from the Natural Sciences and Engineering

Research Council of Canada (NSERC Discovery Grant program; RGPIN-2015-03896) as well as NSERC CREATE (CREATE-463990-2015) and Deutsche Forschungsgemeinschaft DFG (IRTG 2022) for Alberta/Technical University of Munich International Graduate School for Hybrid Functional Materials (ATUMS), as well as Future Energy System (FES) which is supported by the Canada First Research Excellence Fund (CFREF). Canadian Light Source, a national research facility supported by the Canada Foundation for Innovation (CFI), the Natural Sciences and 
Engineering Research Council (NSERC), the National Research Council (NRC), the Canadian

Institutes of Health Research (CIHR), the Government of Saskatchewan, and the University of

Saskatchewan. We also thank the staff at Analytical and Instrumentation Laboratory in the

Department of Chemistry at the University of Alberta for the assistance with FTIR and TGA

analysis. Dr. Shihong Xu and Nancy Zhang of the UofA NanoFab for their assistance with XPS.

Thanks are also conveyed to all Veinot Team members for useful discussions.

\section{Reference}

(1) Polyethylene production U.S. 2019 | Statista https://www.statista.com/statistics/975591/uspolyethylene-production-volume/ (accessed Nov 15, 2020).

(2) Masuda, T.; Matsuki, Y.; Shimoda, T. Stability of Polydihydrosilane Liquid Films on Solid Substrates. Thin Solid Films 2012, 520 (15), 5091-5096.

(3) Beach, J. V.; Loy, D. A.; Hsiao, Y.-L.; Waymouth, R. M. Environmentally Friendly Polysilane Photoresists. In Microelectronics Technology; ACS Symposium Series; American Chemical Society, 1995; Vol. 614, pp 355-366.

(4) Hayase, S. Polysilanes for Semiconductor Fabrication. Prog. Polym. Sci. 2003, 28 (3), 359381.

(5) Motonaga, M.; Nakashima, H.; Katz, S.; Berry, D. H.; Imase, T.; Kawauchi, S.; Watanabe, J.; Fujiki, M.; Koe, J. R. The First Optically Active Polygermanes: Preferential Screw Sense Helicity of Enantiopure Chiral-Substituted Aryl Polygermanes and Comparison with Analogous Polysilanes. J. Organomet. Chem. 2003, 685 (1), 44-50.

(6) Durmaz, Y. Y.; Kukut, M.; Monszner, N.; Yagci, Y. Photoinduced Decomposition of Dibenzoyldiethylgermane: A Photochemical Route to Polygermanes. Macromolecules 2009, 42 (8), 2899-2902.

(7) Mochida, K.; Nagano, S.; Kawata, H.; Wakasa, M.; Hayashi, H. Photodegradation of Thin Films of Polygermanes. J. Organomet. Chem. 1997, 542 (1), 75-79.

(8) Foucher, D. Catenated Germanium and Tin Oligomers and Polymers. In Main Group Strategies towards Functional Hybrid Materials; John Wiley \& Sons, Ltd, 2018; pp 209236.

(9) Weinert, C. S. Germanium: Organometallic Chemistry. In Encyclopedia of Inorganic and Bioinorganic Chemistry; American Cancer Society, 2015; pp 1-18. 
(10) Mochida, K.; Chiba, H. Synthesis, Absorption Characteristics and Some Reactions of Polygermanes. J. Organomet. Chem. 1994, 473 (1), 45-54.

(11) Fa, W.; Zeng, X. C. Polygermanes: Bandgap Engineering via Tensile Strain and Side-Chain Substitution. Chem. Commun. 2014, 50 (65), 9126-9129.

(12) Okano, M.; Toriumi, T.; Hamano, H. Electrochemical Synthesis of Germane-Germane and Germane-Silane Copolymers. Electrochimica Acta 1999, 44 (20), 3475-3482.

(13) Amadoruge, M. L.; Gardinier, J. R.; Weinert, C. S. Substituent Effects in Linear Organogermanium Catenates. Organometallics 2008, 27 (15), 3753-3760.

(14) Kodaira, T.; Watanabe, A.; Ito, O.; Matsuda, M.; Tokura, S.; Kira, M.; Nagano, S.-S.; Mochida, K. Third-Order Nonlinear Optical Properties of Thin Films of Organogermane Homopolymers and Organogermane-Organosilane Copolymers*. Adv. Mater. 1995, 7 (11), 917-919.

(15) Watanabe, A.; Ito, O.; Mochida, K. Photoinduced Electron Transfer from Polygermane to C60 Studied by Laser Flash Photolysis. Organometallics 1995, 14 (9), 4281-4285.

(16) Zaitsev, K. V.; Lam, K.; Zhanabil, Z.; Suleimen, Y.; Kharcheva, A. V.; Tafeenko, V. A.; Oprunenko, Y. F.; Poleshchuk, O. Kh.; Lermontova, E. Kh.; Churakov, A. V. Oligogermanes Containing Only Electron-Withdrawing Substituents: Synthesis and Properties. Organometallics 2017, 36 (2), 298-309.

(17) Huo, Y.; Berry, D. H. Synthesis and Properties of Hybrid Organic-Inorganic Materials Containing Covalently Bonded Luminescent Polygermanes. Chem. Mater. 2005, 17 (1), $157-163$.

(18) Okano, M.; Takeda, K.; Toriumi, T.; Hamano, H. Electrochemical Synthesis of Polygermanes. Electrochimica Acta 1998, 44 (4), 659-666.

(19) Shono, T.; Kashimura, S.; Murase, H. Electroreductive Synthesis of Polygermane and Germane-Silane Copolymer. J. Chem. Soc. Chem. Commun. 1992, No. 12, 896-897.

(20) Reichl, J. A.; Popoff, C. M.; Gallagher, L. A.; Remsen, E. E.; Berry, D. H. RutheniumCatalyzed Demethanative Coupling of HGeMe3: A High Yield Route to Polygermanes. $J$. Am. Chem. Soc. 1996, 118 (39), 9430-9431.

(21) Royen, P.; Schwarz, R. Beiträge Zur Chemie Des Germaniums. XIV. Mitteilung. Die Eigenschaften Des Polygermens (GeH2)x. Z. Für Anorg. Allg. Chem. 1933, 215 (3-4), 295309.

(22) Royen, P.; Rocktäschel, C. Zur Kenntnis niedere Hydride des Siliciums und Germaniums. Z. Für Anorg. Allg. Chem. 1966, 346 (5-6), 279-289.

(23) Choi, K.; Buriak, J. M. Hydrogermylation of Alkenes and Alkynes on Hydride-Terminated Ge(100) Surfaces. Langmuir 2000, 16 (20), 7737-7741.

(24) Javadi, M.; Picard, D.; Sinelnikov, R.; Narreto, M. A.; Hegmann, F. A.; Veinot, J. G. C. Synthesis and Surface Functionalization of Hydride-Terminated Ge Nanocrystals Obtained from the Thermal Treatment of $\mathrm{Ge}(\mathrm{OH}) 2$. Langmuir 2017.

(25) Yu, H.; Helbich, T.; Scherf, L. M.; Chen, J.; Cui, K.; Fässler, T. F.; Rieger, B.; Veinot, J. G. 
C. Radical-Initiated and Thermally Induced Hydrogermylation of Alkenes on the Surfaces of Germanium Nanosheets. Chem. Mater. 2018, 30 (7), 2274-2280.

(26) Helbich, T.; Lyuleeva, A.; Höhlein, I. M. D.; Marx, P.; Scherf, L. M.; Kehrle, J.; Fässler, T. F.; Lugli, P.; Rieger, B. Radical-Induced Hydrosilylation Reactions for the Functionalization of Two-Dimensional Hydride Terminated Silicon Nanosheets. Chem. - Eur. J. 2016, 22 (18), 6194-6198.

(27) Eckerlin, P.; Meyer, H. J.; Wölfel, E. Die Kristallstruktur von CaSn und CaGe. Z. Für Anorg. Allg. Chem. 1955, 281 (5-6), 322-328.

(28) Vogg, G.; Brandt, M. S.; Stutzmann, M. Polygermyne-A Prototype System for Layered Germanium Polymers. Adv. Mater. 2000, 12 (17), 1278-1281.

(29) Helbich, T.; Lyuleeva, A.; Ludwig, T.; Scherf, L. M.; Fässler, T. F.; Lugli, P.; Rieger, B. OneStep Synthesis of Photoluminescent Covalent Polymeric Nanocomposites from 2D Silicon Nanosheets. Adv. Funct. Mater. 2016, 26 (37), 6711-6718.

(30) Bianco, E.; Butler, S.; Jiang, S.; Restrepo, O. D.; Windl, W.; Goldberger, J. E. Stability and Exfoliation of Germanane: A Germanium Graphane Analogue. ACS Nano 2013, 7 (5), 4414-4421.

(31) Cultrara, N. D.; Wang, Y.; Arguilla, M. Q.; Scudder, M. R.; Jiang, S.; Windl, W.; Bobev, S.; Goldberger, J. E. Synthesis of 1T, 2H, and 6R Germanane Polytypes. Chem. Mater. 2018, 30 (4), 1335-1343.

(32) Chen, L.; He, X.; Liu, H.; Qian, L.; Kim, S. H. Water Adsorption on Hydrophilic and Hydrophobic Surfaces of Silicon. J. Phys. Chem. C 2018, 122 (21), 11385-11391.

(33) Kalinkin, A. M.; Kalinkina, E. V.; Zalkind, O. A.; Makarova, T. I. Chemical Interaction of Calcium Oxide and Calcium Hydroxide with $\mathrm{CO} 2$ during Mechanical Activation. Inorg. Mater. 2005, 41 (10), 1073-1079.

(34) Parker, J. H. Raman Scattering by Silicon and Germanium. Phys. Rev. 1967, 155 (3), 712 714.

(35) Weinstein, B. A.; Cardona, M. Second-Order Raman Spectrum of Germanium. Phys. Rev. $B$ 1973, 7 (6), 2545-2551.

(36) Perova, T. S.; Kasper, E.; Oehme, M.; Cherevkov, S.; Schulze, J. Features of Polarized Raman Spectra for Homogeneous and Non-Homogeneous Compressively Strained Ge1-ySny Alloys. J. Raman Spectrosc. 2017, 48 (7), 993-1001.

(37) Thiessen, A. N.; Ha, M.; Hooper, R. W.; Yu, H.; Oliynyk, A. O.; Veinot, J. G. C.; Michaelis, V. K. Silicon Nanoparticles: Are They Crystalline from the Core to the Surface? Chem. Mater. 2019, 31 (3), 678-688.

(38) Plymale, N. T.; Dasog, M.; Brunschwig, B. S.; Lewis, N. S. A Mechanistic Study of the Oxidative Reaction of Hydrogen-Terminated Si(111) Surfaces with Liquid Methanol. $J$. Phys. Chem. C 2017, 121 (8), 4270-4282.

(39) Beekman, M.; Kauzlarich, S. M.; Doherty, L.; Nolas, G. S. Zintl Phases as Reactive Precursors for Synthesis of Novel Silicon and Germanium-Based Materials. Materials 2019, 
$12(7)$.

(40) Schäfer, H.; Eisenmann, B.; Müller, W. Zintl Phases: Transitions between Metallic and Ionic Bonding. Angew. Chem. Int. Ed. Engl. 1973, 12 (9), 694-712.

(41) Park, S. W.; Kaufman-Osborn, T.; Kim, H.; Siddiqui, S.; Sahu, B.; Yoshida, N.; Brandt, A.; Kummel, A. C. Combined Wet and Dry Cleaning of SiGe(001). J. Vac. Sci. Technol. A 2015, 33 (4), 041403.

(42) Palenzona, A.; Manfrinetti, P.; Fornasini, M. L. The Phase Diagram of the Ca-Ge System. J. Alloys Compd. 2002, 345 (1), 144-147.

(43) Fujiki, M.; Kato, M.; Kawamoto, Y.; Kwak, G. Green-and-Red Photoluminescence from Si$\mathrm{Si}$ and $\mathrm{Ge}-\mathrm{Ge}$ Bonded Network Homopolymers and Copolymers. Polym. Chem. 2011, 2 (4), 914-922.

(44) Donovan, T. M.; Spicer, W. E.; Bennett, J. M. Evidence for A Sharp Absorption Edge in Amorphous Ge. Phys. Rev. Lett. 1969, 22 (20), 1058-1061.

(45) Chambreau, S. D.; Zhang, J. GeHx $(\mathrm{X}=0-3)$ and GenHx $(\mathrm{N}=2-7)$ in Flash Pyrolysis of GeH4. Chem. Phys. Lett. 2002, 351 (3), 171-177.

(46) Yu, H.; Helbich, T.; Scherf, L. M.; Chen, J.; Cui, K.; Fässler, T. F.; Rieger, B.; Veinot, J. G. C. Radical-Initiated and Thermally Induced Hydrogermylation of Alkenes on the Surfaces of Germanium Nanosheets. Chem. Mater. 2018, 30 (7), 2274-2280.

(47) Holmberg, V. C.; Korgel, B. A. Corrosion Resistance of Thiol- and Alkene-Passivated Germanium Nanowires. Chem. Mater. 2010, 22 (12), 3698-3703.

For Table of Contents Only:

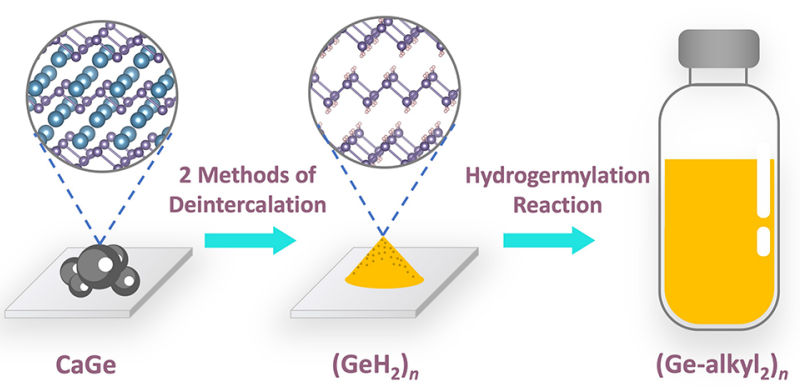

